\title{
Erratum to: Exposure to pesticides and the risk of childhood brain tumors
}

\author{
Kathryn R. Greenop · Susan Peters · Helen D. Bailey • Lin Fritschi • \\ John Attia • Rodney J. Scott • Deborah C. Glass • Nicholas H. de Klerk • \\ Frank Alvaro $\cdot$ Bruce K. Armstrong $\cdot$ Elizabeth Milne
}

Published online: 1 July 2014

(C) Springer International Publishing Switzerland 2014

\section{Erratum to: Cancer Causes Control (2013) 24:1269-1278 \\ DOI 10.1007/s10552-013-0205-1}

After publication, it was noted by the authors that the dataset used for the analyses of occupational exposures in this study accidentally used incomplete occupational histories collected for 94 control fathers and 104 control mothers recruited in 2006, and these control parents were all treated as unexposed to occupational pesticides.

Results in Tables 1-3 and Supplemental Tables 1 and 2 (and the first three rows of Supplemental Table 3) of our published paper were unaffected by this error as they

The online version of the original article can be found under doi: 10 . 1007/s10552-013-0205-1.

K. R. Greenop · H. D. Bailey · N. H. de Klerk · E. Milne $(\bowtie)$ Telethon Kids Institute, University of Western Australia, PO Box 855, West Perth, WA 6872, Australia

e-mail: lizm@ichr.uwa.edu.au

\section{S. Peters}

Western Australian Institute for Medical Research,

University of Western Australia, Perth, WA, Australia

L. Fritschi

Department of Epidemiology and Biostatistics, Curtin

University, Perth, Western Australia, Australia

J. Attia

Centre for Clinical Epidemiology and Biostatistics, School of

Medicine and Public Health, University of Newcastle,

Newcastle, NSW, Australia

J. Attia - R. J. Scott

Hunter Medical Research Institute, School of Biomedical

Sciences, Faculty of Health, University of Newcastle, Newcastle,

NSW, Australia concerned household, rather than occupational pesticide exposure. The conclusions regarding the association with household exposure to professional pest control treatments are consequently unchanged.

However, for occupational exposure, 18 control fathers and 2 control mothers were mistakenly classified as being unexposed to pesticides when they should have been classified as exposed (any time before the child's birth). The analyses presented in Table 4 and parts of Supplemental Table 3 in the published manuscript were re-run using the full dataset to ensure correct exposure assignment ( 5 case fathers and 4 control fathers were additionally excluded from revised occupational analysis due to genuinely missing data). The updated Table 4 and Supplemental Table 3 (occupational estimates only) are presented in this erratum.

\section{R. J. Scott}

Hunter Area Pathology Service, HNEHealth, Newcastle, NSW, Australia

D. C. Glass

Department of Epidemiology and Preventive Medicine,

Monash University, Melbourne, VIC, Australia

F. Alvaro

Department of Paediatric Oncology, John Hunter Children's

Hospital, Newcastle, NSW, Australia

F. Alvaro

School of Medicine and Public Health, University of Newcastle, Callaghan, NSW, Australia

B. K. Armstrong

Sydney School of Public Health, University of Sydney, Sydney, NSW, Australia 
Table 4 Paternal occupational exposure to pesticides and risk of childhood brain tumors

\begin{tabular}{llll}
\hline & $\begin{array}{l}\text { n Cases/ } \\
\text { controls }\end{array}$ & $\mathrm{OR}^{\mathrm{a}}$ & $95 \% \mathrm{CI}$ \\
\hline $\begin{array}{l}\text { Not exposed any time before the } \\
\text { pregnancy }\end{array}$ & $201 / 680$ & 1.0 & Referent \\
$\begin{array}{l}\text { Exposed any time before the } \\
\text { pregnancy }\end{array}$ & $32 / 103$ & 1.07 & $0.68,1.68$ \\
$\begin{array}{l}\text { Exposed in the year before } \\
\text { pregnancy }\end{array}$ & $13 / 37$ & 1.11 & $0.55,2.23$ \\
\end{tabular}

a Adjusting for matching variables: child age, sex, state of residence, year of birth group, parental education, child's ethnicity, fathers age, maternal and paternal occupational diesel exhaust exposure

Our original results for paternal occupational pesticide exposures were imprecise, and we concluded that these results were less clear than for household exposure but suggestive of a positive association. The revised ORs for paternal occupational exposure to pesticides are attenuated and offer little or no evidence of an increased risk. Our imputation analysis (Supplemental Table 3) still shows that these results were unlikely to be affected by bias due to missing job histories.

The sentence in the abstract concerning fathers' occupational exposure should now read

"The OR for paternal occupational exposure in the year before the child's conception was 1.11 (95\% CI: 0.55 ,
2.23)." There were still too few occupationally exposed mothers to analyse ( 2 cases/16 controls).

The authors offer their sincere apologies for any confusion that may have caused.

Supplementary Table 3: Paternal occupational pesticide exposure and the risk of childhood brain tumors, with controls with missing exposure data imputed

Exposure \% from available controls with IRSD in the same range as those controls missing data

\begin{tabular}{lll}
\hline $\begin{array}{l}\text { \% exposure applied } \\
\text { to missing controls }\end{array}$ & $\mathrm{OR}^{\mathrm{a}}$ & $95 \% \mathrm{CI}$ \\
\hline $13.3 \%$ & 1.08 & $0.72,1.62$ \\
& & \\
\hline $.9 \%$ & 1.16 & $0.62,2.14$ \\
& & \\
\hline
\end{tabular}

Paternal occupational exposure any time before birth

Paternal occupational exposure in the year before pregnancy

${ }^{a}$ With 1,467 controls, those with missing data imputed in $\mathrm{R}$ with ORs adjusted for child age, sex, state, year of birth group and IRSD. IRSD: Index of relative socioeconomic disadvantage, OR: odds ratio; CI: confidence interval 